\title{
Stimulus-Specific Adaptation Occurs in the Auditory Thalamus
}

\author{
Lucy A. Anderson, ${ }^{1}$ G. Björn Christianson, ${ }^{1}$ and Jennifer F. Linden ${ }^{1,2}$ \\ ${ }^{1}$ Ear Institute, University College London, London WC1X 8EE, United Kingdom, and ²Department of Neuroscience, Physiology and Pharmacology, \\ University College London, London WC1E 6BT, United Kingdom
}

Neurons in the primary auditory cortex respond less strongly to a commonly occurring "standard" tone than to the same tone when it is rare or "deviant." This phenomenon, called "stimulus-specific adaptation" (SSA), has been proposed as a possible single-neuron correlate of the mismatch negativity, a cortical evoked potential associated with stimulus novelty. Previous studies in cat did not observe SSA in single neurons in the auditory thalamus. However, these reports did not differentiate between the auditory thalamic subdivisions and did not examine the effects of changing the stimulus presentation rate. To explore the possibility of thalamic SSA more completely, we recorded extracellularly from 30 single units and 22 multiunit clusters in the ventral, medial, and dorsal subdivisions of the mouse medial geniculate body (MGB), while presenting the anesthetized animals with sequences of standard and deviant tones at interstimulus intervals of 400, 500 and $800 \mathrm{~ms}$. We found SSA in the auditory thalamus at all three stimulus presentation rates, primarily in the medial subdivision but to a lesser degree also in the ventral MGB. Thalamic SSA was evident from the earliest onset of tone-evoked activity, although the latencies of responses to standard and deviant tones were not significantly different. Together with related findings of SSA in neurons of the "belt" regions of the inferior colliculus, these results demonstrate that SSA is present at subcortical levels, primarily in but not restricted to the nonlemniscal auditory pathway.

\section{Introduction}

Stimulus-specific adaptation (SSA), or frequency-selective habituation to commonly occurring tones, was recently described in the cat primary auditory cortex and proposed as a possible neuronal correlate of the mismatch negativity (Ulanovsky et al., $2003,2004)$. The authors found no evidence for SSA in the main nucleus of the auditory thalamus [the medial geniculate body (MGB)] and suggested that SSA might, therefore, be a cortical phenomenon. However, those thalamic studies used only stimuli presented at an interstimulus interval of $800 \mathrm{~ms}(\sim 1.25$ stimuli/ $\mathrm{s}$ ), and the locations of the recordings among the auditory thalamic subdivisions were not specified. We wondered if SSA might be observed in the MGB only for stimuli presented at faster rates or only in particular thalamic subdivisions. To address these possibilities, we recorded single-unit and multiunit activity in the medial, ventral, and dorsal subdivisions of the mouse MGB, using a stimulus identical to that of Ulanovsky et al. (2004) but with interstimulus intervals of 400, 500, and $800 \mathrm{~ms}$. Preliminary versions of this study have appeared previously in abstract form (Linden and Anderson, 2008; Anderson et al., 2009a).

Received Feb. 16, 2009; revised April 9, 2009; accepted May 8, 2009

This work was supported by grants from the Gatsby Charitable Foundation (GAT2579 and GAT2623) and Deafness Research UK (412:UEl:JL).

Correspondence should be addressed to Dr. Jennifer F. Linden, Ear Institute, University College London, 332 Gray's Inn Road, London WC1X 8EE, UK. E-mail: j.linden@ucl.ac.uk.

DOI:10.1523/JNEUROSCI.0793-09.2009

Copyright $\odot 2009$ Society for Neuroscience $\quad$ 0270-6474/09/297359-05\$15.00/0

\section{Materials and Methods}

Animals. Ten adult male CBA/Ca mice, 6-24 weeks of age, were used as subjects in this experimental study. Mice were anesthetized with ketamine and medetomidine, prepared for recording and monitored using procedures similar to those described by Linden et al. (2003). A craniotomy $\sim 3 \mathrm{~mm}$ in diameter, centered $2 \mathrm{~mm}$ lateral to midline and $3 \mathrm{~mm}$ caudal to bregma, was performed on the left-hand side, enabling vertical access to the MGB. Extracellular recordings were made across all MGB subdivisions using custom-made tungsten-in-glass electrodes (tip sizes, 10-20 $\mu \mathrm{m}$; impedance typically, 1-2 M $\Omega$ ) (Bullock et al., 1988). All experiments were performed in accordance with the United Kingdom Animal (Scientific Procedures) Act of 1986.

Auditory stimuli. Free-field auditory stimuli were directed at the animal's right (contralateral) ear, and a sound-attenuating plug was placed in the left ear. Before the start of each experiment, acoustic stimuli were calibrated with a microphone positioned as close as possible to the opening of the animal's auditory canal. The sound system frequency response was flat to within $\pm 1.5 \mathrm{~dB}$ from 2 to $90 \mathrm{kHz}$. Responses to tone pips of variable frequency and intensity were used to estimate the frequencyresponse area and characteristic frequency $(\mathrm{CF})$ for each $\mathrm{MGB}$ neuron. If the neuron showed a clear response to tonal stimuli (as indicated by the frequency response area), stimulus-specific adaptation was assessed using two frequencies ( $\mathrm{f} 1$ and $\mathrm{f} 2$ ) presented in a "switching oddball" paradigm exactly as used for analysis of SSA in cat auditory cortex (Ulanovsky et al., 2004). Briefly, the switching oddball paradigm consisted of blocks of 800 trials, 20 repetitions of a "frozen" sequence of 40 trials. For the first 20 trials, the frequencies $\mathrm{f} 1$ and $\mathrm{f} 2$ occurred with probabilities 0.8 and 0.2 , respectively; in the second 20 trials, the probabilities were reversed. Individual tones were $200 \mathrm{~ms}$ long, with a cosine-squared rise/fall time of 5 $\mathrm{ms}$, and were presented at $20 \mathrm{~dB}$ SPL above threshold at CF. The interval between successive tones in the stimulus [interstimulus interval (ISI)] was either 400, 500, or $800 \mathrm{~ms}$. As in Ulanovsky et al. (2004), the frequencies $\mathrm{f} 1$ and $\mathrm{f} 2$ were separated by no more than 0.5 octaves, with the central 
frequency $(\mathrm{f} 2 \times \mathrm{f} 1)^{1 / 2}$ close to the neuron's characteristic frequency. This condition was equivalent to the condition $(\mathrm{f} 2-\mathrm{f} 1) /(\mathrm{f} 2 \times \mathrm{f} 1)^{1 / 2}=0.37$, described by Ulanovsky et al. $(2003,2004)$.

Histology. Localization of recording sites to each of the three major subdivisions within the MGB was accomplished by reconstruction of electrolytic lesions and recording tracks from brain sections stained for cytochrome oxidase. Histological procedures were exactly as described by Anderson et al. (2009b).

Data analysis. A neuronal SSA index ("neuronal SI") was defined as in Ulanovsky et al. $(2003,2004)$ : neuronal SI $=[\operatorname{dev}(\mathrm{f} 1)+\operatorname{dev}(\mathrm{f} 2)-$ $\operatorname{std}(\mathrm{f} 1)-\operatorname{std}(\mathrm{f} 2)] /[\operatorname{dev}(\mathrm{f} 1)+\operatorname{dev}(\mathrm{f} 2)+\operatorname{std}(\mathrm{f} 1)+\operatorname{std}(\mathrm{f} 2)]$, where $\operatorname{dev}(\mathrm{f} 1)$ is the average neuronal response to $\mathrm{f} 1$ when $\mathrm{f} 1$ is deviant, $\operatorname{std}(\mathrm{f} 1)$ is the average response to $\mathrm{f} 1$ when $\mathrm{f} 1$ is standard, and likewise for $\operatorname{dev}(\mathrm{f} 2)$ and $\operatorname{std}(\mathrm{f} 2)$. For each neuron, the significance of this neuronal SI value was assessed by comparing the actual value to the distribution expected under the null hypothesis of no difference between responses to standard and deviant stimuli (approximated using a Monte Carlo simulation with 10,000 randomizations of the assignments of tone responses to standard or deviant stimuli). A given neuronal SI value was considered significant if its probability in this randomization test was $<0.01$. Using a similar Monte Carlo approach, we also tested for significant differences in firstspike latency (defined as the mode of the first spike times after tone onset) between the standard and deviant conditions.

\section{Results}

We recorded from 30 single units and 22 multiunits in the MGB of 10 mice. Similar trends were observed among single units and multiunits separately, so results are reported here for pooled single-unit and multiunit data. All neurons responded to pure tones and showed similar responses to both frequencies chosen for the switching oddball stimuli. Responses were generally transient, consisting of a short burst of spikes after tone onset. Neuronal firing rate was, therefore, analyzed in the 0-60 ms interval after tone onset, which encompassed the entire response evident in population peristimulus time histograms (PSTHs) (Fig. 1). Larger analysis windows produced qualitatively similar results.

Population PSTHs averaged over all recordings in the MGB demonstrated that when a tone was presented as a standard (Fig. $1 A$, blue), it evoked a weaker response than when it was presented as the deviant stimulus (Fig. $1 \mathrm{~A}$, red). This stimulus-specific adaptation was evident for switching oddball stimuli with interstimulus intervals of 400, 500, and even 800 ms. Analysis of neuronal SIs revealed significant SSA at all three stimulus presentation rates (Fig. $1 B$ ). The median neuronal SI was significantly positive (indicating greater response to deviant than standard tones) at all interstimulus intervals tested (sign-rank test, $p<0.01$ in all cases). Moreover, individual neuronal SIs were significant in $11-16 \%$ of the population, depending on presentation rate $(p<0.01$ by a randomization test; see Materials and Methods). Thus, at interstimulus intervals of 400, 500, and even $800 \mathrm{~ms}$, there was significant SSA in the mouse MGB both at the population level and in a subpopulation of individual neurons.

To better illustrate the time course and magnitude of the SSA, Figure $1 C$ presents, on an expanded timescale, population PSTHs calculated only for the individually significant neurons. Neurons in this subpopulation responded strongly to the $\mathrm{f} 1$ and $\mathrm{f} 2$ stimuli (compare $y$-scale in Fig. $1 C$ to that in Fig. $1 A$ ) and had a large difference signal (for example, at $500 \mathrm{~ms}$ ISI, the difference signal was approximately half the magnitude of the population response to the standard tone). As is also evident in Figure $1 \mathrm{~A}$ for the entire population, the latency of the difference signal in Figure $1 C$ was similar to the latency of the standard and deviant population responses themselves at all ISIs, indicating that SSA emerged very early in the population response. At the single neuron level, there was no difference between the first-spike latencies to either the standard or deviant conditions, regardless of the interstimulus interval ( $p>0.05$ in all cases using the randomization test; see Materials and Methods). However, over the period 5-15 ms after tone onset, which encompassed the earliest tone responses, the mean difference between responses to deviant and standard tones was significantly positive for 500 and $800 \mathrm{~ms}$ ISIs ( $t$ test, $p<0.01$ ) and positive although not significantly so for $400 \mathrm{~ms}$ ISI.

To determine whether the prevalence of SSA differed between MGB subdivisions, we localized recording sites to the medial $(N=$ $20)$, ventral $(N=19)$, or dorsal $(N=13)$ subdivisions by reconstructing electrode tracks in brain sections stained for cytochrome oxidase (supplemental Fig. 1, available at www.jneurosci.org as supplemental material). The range of characteristic frequencies of recorded neurons was similar for the three MGB subdivisions. However, population histograms revealed that stimulus-specific adaptation was most pronounced in the medial MGB population, at all ISIs tested (supplemental Fig. 2, available at www.jneurosci.org as supplemental material).

This finding was confirmed by analysis of neuronal SI histograms (Fig. 2). Median neuronal SI was significantly positive in the medial MGB (sign-rank test, $p<0.01$ at all three ISIs), and $\sim 20 \%$ of medial MGB neurons had neuronal SIs that were individually significant at the $p<0.01$ level (Fig. $2 A$ ). In the ventral MGB, $\sim 10 \%$ of the neurons had significant neuronal SIs $(p<$ $0.01)$, but the median neuronal SI for the population was not significantly different from zero (sign-rank test, not significant for any ISIs). A smaller sample of neurons from the dorsal MGB showed no significant SSA at either the population level or in individual neurons (Fig. 2C). Thus, stimulus-specific adaptation occurred primarily in the medial MGB subdivision and was similar for switching oddball stimuli with ISIs of 400, 500, and $800 \mathrm{~ms}$ (for further analysis of the effects of ISI, see supplemental Fig. 3, available at www.jneurosci.org as supplemental material).

\section{Discussion}

Stimulus-specific adaptation occurred in the mouse MGB at all stimulation rates tested, even the relatively slow $800 \mathrm{~ms}$ ISI used in previous studies that had reported no SSA in the cat MGB (Ulanovsky et al., 2003, 2004). However, in the mouse MGB, the SSA was evident primarily in the medial subdivision; the effect was much less pronounced in the ventral subdivision and was not detected in a smaller sample of neurons from the dorsal MGB. Although we observed SSA in the larger ventral MGB, it was not significant at the population level; thus, it may be that thalamic SSA was not observed in the cat MGB, since the blind sampling strategy may not have included a sufficiently large population from the medial MGB. Alternatively, it may be that SSA is an exclusively cortical phenomenon in the cat but not in rodents.

The medial subdivision of the MGB is part of the nonlemniscal auditory pathway and receives the majority of its projections from the belt regions of the inferior colliculus (Winer, 1992). In addition to auditory projections, the medial MGB has strong connections with the amygdala (LeDoux et al., 1990) and somatosensory pathways among other areas (Rouiller et al., 1989; Winer, 1991, 1992). The medial MGB is also known to contain neurons with short-latency responses to acoustic stimuli (Anderson et al., 2006). Based on these observations, it has been suggested that the medial MGB may mediate alerting responses to acoustic stimuli. Stimulus-specific adaptation, which essentially amounts to habituation of auditory responses to frequently occurring sounds, may play an important role in these alerting re- 
$400 \mathrm{~ms}$ ISI

A

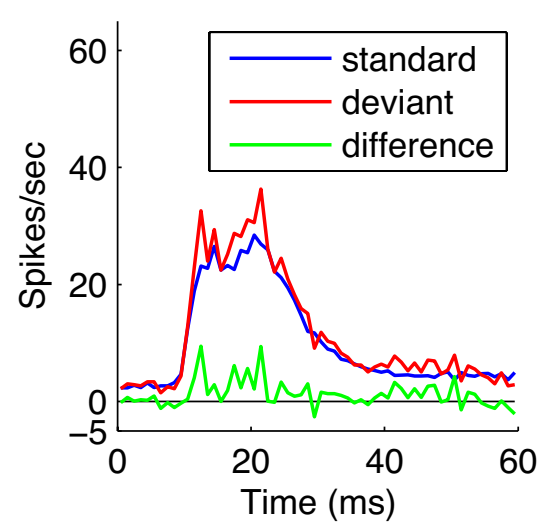

B

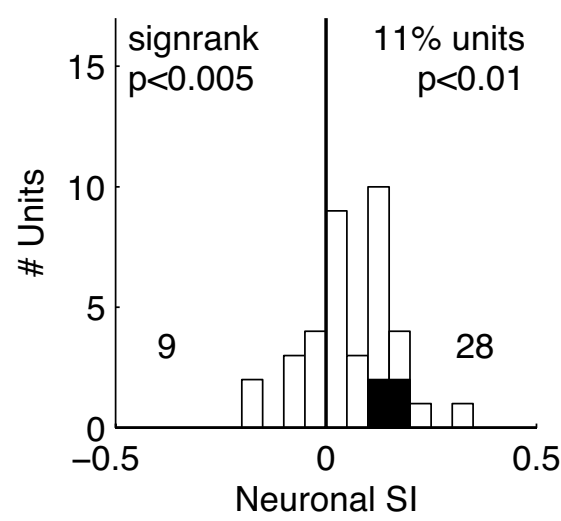

C

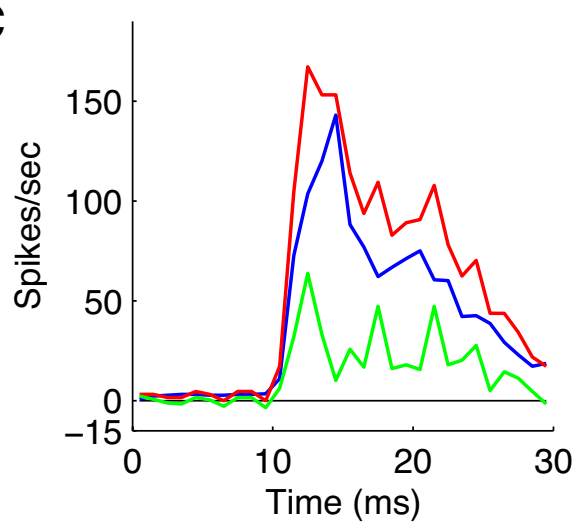

$500 \mathrm{~ms} I S I$
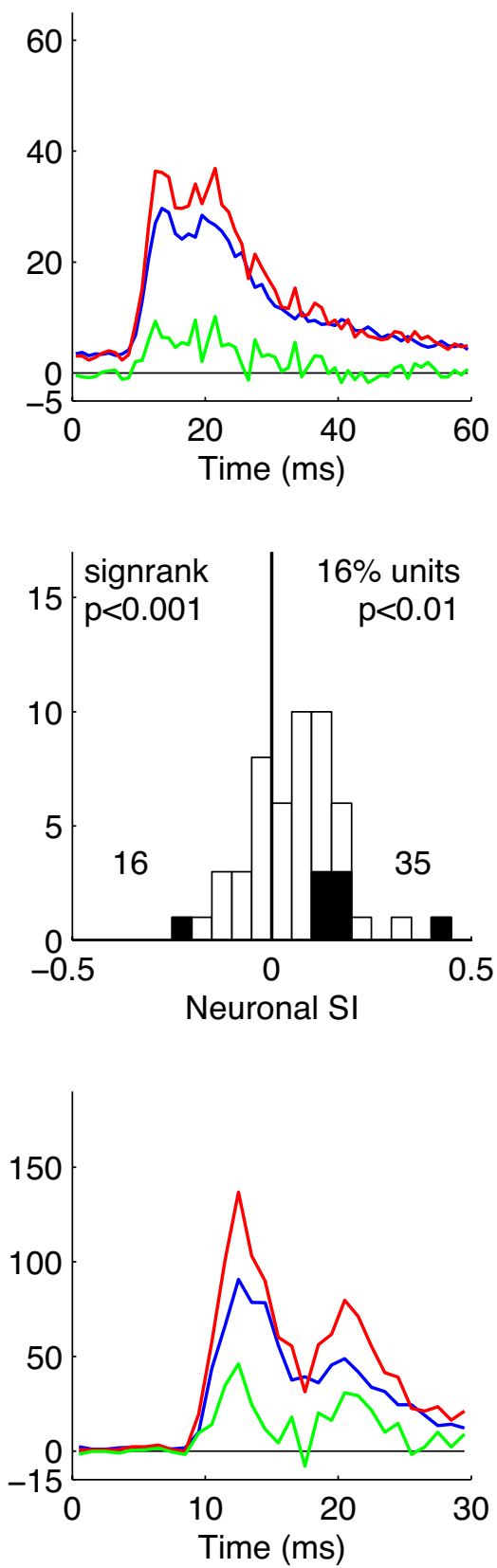

$800 \mathrm{~ms}$ ISI
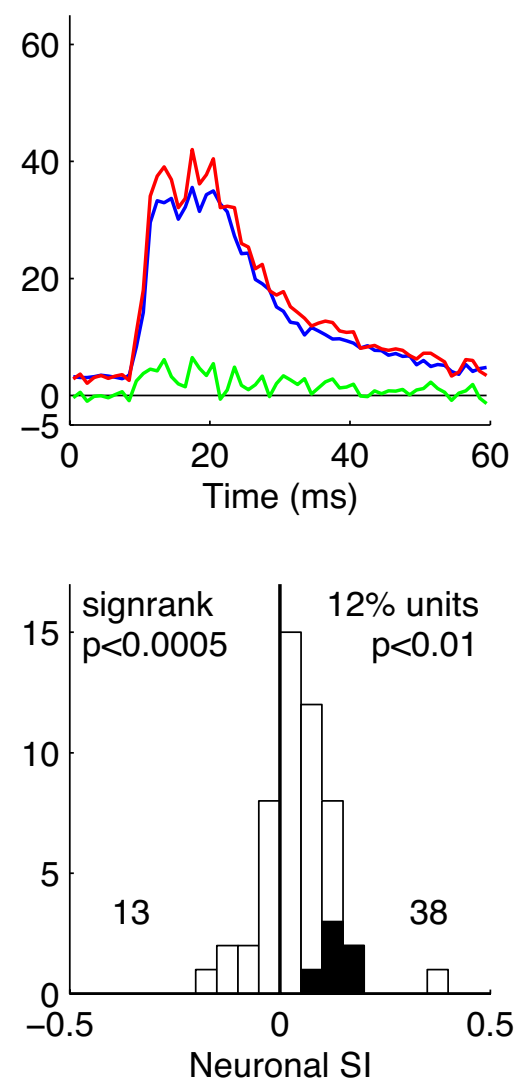

Figure 1. Stimulus-specific adaptation in the mouse MGB. A, Population PSTHs averaged over all MGB recordings, showing responses to switching oddball stimuli with interstimulus intervals of 400 (left), 500 (middle), or $800 \mathrm{~ms}$ (right). Each plot compares the average neuronal response to tones presented as standards (blue) with the average response to the same tones presented as deviants (red); the average difference in response is also shown (green). $\boldsymbol{B}$, Histograms of neuronal stimulus-specific adaptation indices (neuronal SIs) for tone responses recorded using switchingoddball stimuli with interstimulus intervals of 400 (left), 500 (middle), or $800 \mathrm{~ms}$ (right). Recordings with individually significant neuronal Sls ( $p<0.01$ ) are indicated with black bars. Numbers and text within each plot indicate the following: (bottom left and right) the number of recordings with neuronal Sls above and below zero; (top left) the result of a sign-rank test of the hypothesis that the median neuronal SI was zero; and (top right) the percentage of recordings for which the neuronal SI was individually significant by a randomization test ( $p<0.01$ ). C, Population PSTHs showing responses averaged over only those MGB recordings for which the neuronal SIs were significant $(p<0.01)$ and with an expanded timescale around response onset. Conventions as in $A$ but note different $x$-scale and $y$-scale.

sponses, by suppressing responsiveness to common sounds and thereby highlighting low-probability, more unexpected sounds.

Like SSA in the cat auditory cortex (Ulanovsky et al., 2003, 2004), SSA in the mouse MGB was evident through the entire duration of the neuronal response. Mouse MGB responses were usually very transient (as are MGB responses in other species) (for review, see De Ribaupierre, 1997), so it was not possible to determine whether SSA in mouse MGB affected sustained activ- ity $>60 \mathrm{~ms}$ after tone onset, as demonstrated in the auditory cortex (Ulanovsky et al., 2003, 2004). However, the SSA of the transient portions of the responses in the mouse MGB resembled those in the cat auditory cortex [compare Fig. $1 A$ with Ulanovsky et al. (2003), their Fig. 2A,B]. SSA in mouse MGB differed from the SSA reported in cat auditory cortex in that individual neuronal SI values for mouse MGB neurons were $<0.5$, whereas in the cat auditory cortex, a small subpopulation of neurons were re- 

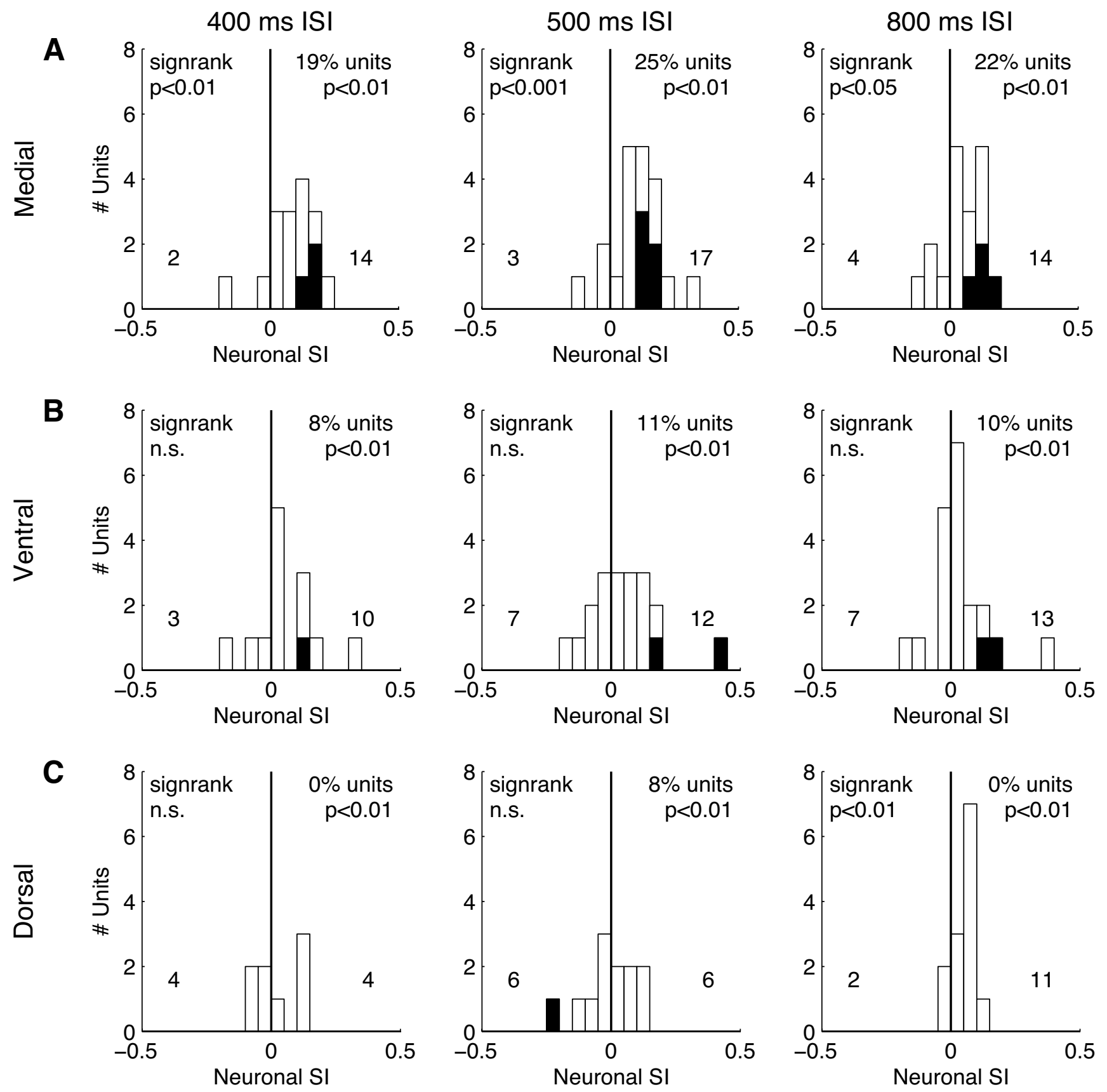

Figure 2. Stimulus-specific adaptation occurs primarily in the medial subdivision of the mouse MGB. Plotting conventions as in Figure $1 B$. Histograms show neuronal SIs for recordings in the medial $(\boldsymbol{A})$, ventral $(\boldsymbol{B})$, and dorsal $(\boldsymbol{C})$ MGB, for each of the three different interstimulus intervals.

ported to have neuronal SI values exceeding 0.6 (Ulanovsky et al., 2003, 2004). Recent conference abstracts have reported SSA in the MGB of two other rodent species; the neuronal SI values reported here are comparable with those observed in the gerbil (Baeuerle et al., 2009) but lower than those reported in the rat (Antunes et al., 2009). These differences in neuronal SI values for MGB neurons in mouse, gerbil, and rat may reflect species differences or could have arisen from variations in experimental protocols, including differences in the type of anesthesia or the neuronal search strategies used.

Stimulus-specific adaptation has a relatively short latency $(<15 \mathrm{~ms})$ across the auditory thalamic population in the mouse. In fact, the divergence between the mean response to tones presented as standards and the mean response to the same tones presented as deviants was evident from the earliest onset of toneevoked activity (Fig. 1A,C). This finding suggests that thalamic SSA is unlikely to arise through stimulus-driven modulation of thalamic responses by cortical feedback, which would be expected to introduce a delay of at least a few milliseconds between the onset of tone responses and the onset of SSA. Fast-onset thalamic SSA could arise through longer-term cortical modulation of thalamic excitability or the excitability of other subcortical auditory areas; however, we observed no difference in first-spike latencies between standard and deviant conditions $(p>0.05)$. Alternatively, SSA might be a general property of many neurons in the auditory system, particularly within the nonlemniscal pathway. SSA is also known to occur in neurons of the nonlemniscal belt regions of the inferior colliculus (Pérez-González et al., 
2005; Reches and Gutfreund, 2008; Malmierca et al., 2009). Together with the data on thalamic SSA presented here, these findings suggest that whatever its origin, SSA is an important feature of subcortical nonlemniscal auditory processing.

\section{References}

Anderson LA, Malmierca MS, Wallace MN, Palmer AR (2006) Evidence for a direct, short latency projection from the dorsal cochlear nucleus to the auditory thalamus in the guinea pig. Eur J Neurosci 24:491-498.

Anderson LA, Christianson GB, Linden JF (2009a) Stimulus-specific adaptation occurs in neurons of the medial but not ventral auditory thalamus. Assoc Res Otolaryngol Abstr 114:40.

Anderson LA, Christianson GB, Linden JF (2009b) Mouse auditory cortex differs from visual and somatosensory cortices in the laminar distribution of cytochrome oxidase and acetylcholinesterase. Brain Res 1252:130-142.

Antunes F, Covey E, Malmierca MS (2009) Contribution of the thalamus to detection of novel sounds: is there stimulus specific adaptation in the medial geniculate body of the rat? Assoc Res Otolaryngol Abstr 102:35.

Baeuerle P, von der Behrens W, Gaese B, Koessl M (2009) Stimulus-specific adaptation in the auditory thalamus of the Mongolian gerbil. Assoc Res Otolaryngol Abstr 110:38.

Bullock DC, Palmer AR, Rees A (1988) A compact and easy to use tungsten in glass microelectrode manufacturing workstation. Med Biol Eng Comput 26:669-672.

De Ribaupierre F (1997) Acoustical information processing in the auditory thalamus and cerebral cortex. In: The central auditory system (Ehret G, Romand R, eds), pp 317-398. Oxford: Oxford UP.

LeDoux JE, Farb C, Ruggiero DA (1990) Topographic organization of neurons in the acoustic thalamus that project to the amygdala. J Neurosci 10:1043-1054.
Linden JF, Anderson LA (2008) Stimulus-specific adaptation occurs in neurons of the medial but not ventral auditory thalamus. Soc Neurosci Abstr 34:664.27.

Linden JF, Liu RC, Sahani M, Schreiner CE, Merzenich MM (2003) Spectrotemporal structure of receptive fields in areas AI and AAF of mouse auditory cortex. J Neurophysiol 90:2660-2675.

Malmierca MS, Cristaudo S, Pérez-González D, Covey E (2009) Stimulusspecific adaptation in the inferior colliculus of the anesthetized rat. J Neurosci 29:5483-5493.

Pérez-González D, Malmierca MS, Covey E (2005) Novelty detector neurons in the mammalian auditory midbrain. Eur J Neurosci 22:2879-2885.

Reches A, Gutfreund Y (2008) Stimulus-specific adaptations in the gaze control system of the barn owl. J Neurosci 28:1523-1533.

Rouiller EM, Rodrigues-Dagaeff C, Simm G, De Ribaupierre Y, Villa A, De Ribaupierre F (1989) Functional organization of the medial division of the medial geniculate body of the cat: tonotopic organization spatial distribution of response properties and cortical connections. Hear Res 39:127-142.

Ulanovsky N, Las L, Nelken I (2003) Processing of low-probability sounds by cortical neurons. Nat Neurosci 6:391-398.

Ulanovsky N, Las L, Farkas D, Nelken I (2004) Multiple time scales of adaptation in auditory cortex neurons. J Neurosci 24:10440-10453.

Winer JA (1991) Anatomy of the medial geniculate body. In: Neurobiology of hearing - the central auditory system (Altschuler RA, Bobbin RP, Clopton BM, Hoffman DW, eds), pp 293-335. New York: Raven.

Winer JA (1992) The functional architecture of the medial geniculate body and the primary auditory cortex. In: The mammalian auditory pathway: neuroanatomy (Webster DB, Popper AN, Fay RR, eds), pp 222-409. New York: Springer. 